\title{
Test planning based on ontological models constructed from product usage profiles
}

\author{
Lucas Barboza Zattar Paganin, Milton Borsato, Juliana Schmidt, Augusto Domingos, Roberto Shigueru Sato \\ Universidade Tecnológica Federal do Paraná - UTFPR \\ e-mails: lucas.paganin@alunos.uffrr.edu.br; borsato@uffpr.edu.br; iscmidt@alunos.uffpr.edu.br; \\ augustodomingos@alunos.utfpr.edu.br; robertosato@uffpr.edu.br
}

\begin{abstract}
Design for Reliability (DfR) is such an approach that can be referred as a set of activities that attempts to ensure the reliability of a product throughout all stages of its lifecycle. Recent research works on the subject, as well as common practices by industry, have revealed no cases of implementation of DfR in the initial stages of new product development, such as the definition of test plans based on product usage profiles. Therefore, the main goal of this research is to elaborate a method, based on an ontological model, that allows the determination of the most appropriate test plan, by considering usage characteristics of products. In order to develop this method, the Design Science Research methodological framework was used. Achieved results show that the proposed solution is an efficient and easy-to-use method, which potentially improves product reliability throughout the product lifecycle.
\end{abstract}

Keywords: design for reliability, new product development, test planning, ontology, product lifecycle.

\section{Introduction}

It all starts with creativity, creativity techniques are one-off tools that can be used to induce the creative potential of an individual or a group of individuals for the purpose of solving problems or generating ideas in the development of new products and services (LEOPOLDINO et al., 2016).

In recent decades, the increase of focus on consumer satisfaction has made studies on product reliability more prominent. According to Waghmode and Patil (2016, p. 414-429), reliability can be defined as

"[...] the probability that a component, device, system or process will operate without failures for a given period of time, if correctly used in a previously specified environment".

The lack of reliability in a product has a number of unwanted consequences in terms of safety, competitiveness, cost of maintenance and repair, and brand reputation, among others (ASHRAFI; DAVOUDPOUR, 2019).

According to Yang et al. (2011), reliability-oriented activities should be considered in the early stages and throughout the New Product Development (NPD) process. Hence, Design for Reliability (DfR) arises as an alternative approach for assisting NPD professionals in the identification of prototyping and testing problems, aiming at the reduction of lifecycle cost, field failure rates and the time to market. Crowe and Feinberg (2001, p. 14) define DfR as a set of practices that is designed to improve the reliability of a product. This approach ranges from understanding the product requirements and customers' needs to monitoring the reliability of the developed product.

Critical components that may be linked to the reliability of a product should be studied, understood, and considered when designing a product and determining DfR-related activities. According to Kuei-Chen, Yeu-Shiang and I-Chi (2019), such elements may come from the internal composition of the product (e.g. parts, devices and even software that may influence the operation of the product) or from external factors (e.g. environmental conditions in which the product may be used, such as humidity, climate, lighting and others). In this way, a more detailed study of a product's components, as well as an investigation of the item's interactions with its location and usage profile, can significantly improve its reliability (BRALLA, 1996, p. 170).

Currently, industries have been undergoing several transformations, in which the real and virtual world have been combined following the principle of the Internet of Things (IoT). Such a concept has stimulated a new industrial age known as Industry 4.0. In this new industrial context, production is highly flexible and customizable and factories are related to the concepts of Smart Manufacturing (SM) (SHROUF et al., 2014; NUÑEZ; BORSATO, 2017; LU, 2017; ZHONG et al., 2017; DA XU; XU, LI, 2018). Trappey et al. (2017) state that one of the ways to implement the concepts of the IoT and SM is through ontologies, 
which can be used to manage information and to transmit and process protocols, since they are designed to represent knowledge related to a specific domain.

In order to gather some practical information about the concepts of reliability in the NPD, meetings and interviews with representatives of a global agricultural machinery manufacturing company located in the southern region of Brazil were conducted. A lack of in-depth knowledge related to the test planning used to evaluate the reliability of some products was noticed, because their expected field usage profile was barely considered. This fact corroborates with the gap described in the literature review. It was observed that none of the most recent studies presents the development of an ontological model in order to investigate the influence of critical components and field usage profile on the product's test planning.

The purpose of the present work, therefore, is to construct an ontological model that can assist NPD professionals in the design of the most appropriate test plan to a specific item. As a consequence, this model will ensure the implementation of DfR principles in the initial stages of a product's creation process. Information related to critical factors and product usage is investigated and modeled in the form of an ontology.

This article is organized in five sections. Section 2 brings a literature review on product reliability in the NPD. Section 3 introduces methodological aspects regarding the application of DSR framework in the present proposal. Section 4 presents the development of the ontological model. Section 5 brings the most relevant contributions of the present work as final considerations.

\section{Literature review}

In order to establish the reliability of a product, DfR activities should be implemented from the early stages of the NPD process. Such activities, coupled with efficient testing planning, ensure that the new product is reliable through its entire lifecycle (TANASIJEVIC et al., 2019). The knowledge concerning the products' specifications and usage must be considered to accomplish an efficient test planning. Therefore, ontologies may be useful tools to determine the most appropriate tests aiming at the improvement in reliability. In this context, the use of ontological models in the implementation of DfR-related activities in the first stages of the NPD indicates that concepts of Smart Manufacturing are applied.

\subsection{DfR in the context of the NPD process}

Reliability of a new given item is a must for its acceptance in the market. Thus, Engineering teams that support the NPD in all stages are demanded to overcome this challenge. Reliability-oriented teams must perform three fundamental tasks, altogether: DfR activities, reliability verification and physical product analysis (CROWE; FEINBERG, 2001, p.14).

Pahl and Beitz (2013, p. 15) define a workflow to be adopted during the NPD process. The first phase refers to the information acquisition about requirements to be considered and limitations. The following phase is associated with the definition of specifications, when the conceptual design of a product is being elaborated. This phase is subdivided into several steps which should be followed to achieve a successful conceptual design. The method developed in the present work should be applied in these first two phases, since it is more straightforward and reasonable to implement activities aimed at improving the quality and reliability in the early stages of the NPD (PAHL; BEITZ, 2013; BOUNCKEN et al., 2018; CUI; WU, 2017). In addition, the first two phases comprehend the optimization of the proposed principles from the specifications. In these phases, the product is still an abstraction, then, any changes in its design will result in less technical and economic consequences when compared to changes made in the later phases.

Ontological modelling may be considered an assisting tool for implementing DfR principles in the NPD process. Such tool allows knowledge capture and, through an ontology editor and inference engines, useful and valid information about the domain can be obtained. In this way, relevant factors about NPD and reliability principles should be considered in order to generate more knowledge about the best policies to be adopted. The use of ontologies for this purpose has not been observed in the most recent studies.

\subsection{Ontologies}

The term ontology can be used with divergent meanings in different situations. The most radical disagreement appears between the more traditional and recognized philosophical sense and the computational sense that has recently emerged among engineers (STAAB; STUDER, 2009 , p. 1). Ontologies have been widely used in Knowledge-base Engineering (KBE) since 1990, in order to settle the needs associated with the sharing and reuse of information.

According to the computational sense, ontologies seek to capture consensual knowledge about a given subject in a generic and formal way, making such information shared and reused by software and by people (CORCHO et al., 2004). Gruber (1993, p. 199-220) defines ontologies as "an explicit specification of conceptualization”. Gangemi (2005, p.262276 ) define the term ontology as "[...] a diagram whose nodes and arcs represent concepts [...]". This perspective of ideas is a representation of metadata encompassing terms grouped into categories, taxonomies, and axioms (BILGIN; DIKMEN; TALAT BIRGONUL, 2014). 
Ontologies define a common vocabulary for researchers who need to share information about a given domain, including definitions of basic concepts and their relationships. Noy and MacGuinness (2001) state that the main objectives for ontology creation within a domain are:

- Sharing common knowledge among people or software;

- Allowing the reuse of knowledge;

- Clarifying some assumptions and considerations;

- Unravelling operational knowledge from general knowledge; and

- Exploring knowledge.

The process of creating an ontology demands the use of tools to represent and edit the model. The main tools available are Onto Studio, Apollo, Top Braid Composer Free Edition, Swoop and Protégé, which cover a wide variety of ontology development processes. In this work, the selected tool was Protégé, version 5.2.0 (STANFORD CENTER FOR BIOMEDICAL INFORMATICS RESEARCH, 2017), which is available online and free of charge from Stanford University. This editor was chosen because of its massive base of users and discussion forums, availability of plug-ins and extensions, and ease-of-use. In addition, it has a customizable interface capable of adapting to different formal languages and an efficient plug-in architecture capable of integrating with other applications (GENNARI et al., 2003).

Inference Engines (IE) are used in Protégé to reveal new knowledge based on what is already known. In agreement with AKERKAR; SAJJA (2010, p. 36), an IE is a program capable of manipulating existing knowledge. This is executed according to the needs to make decisions and the actions are performed using standard combination techniques which search for conclusions. Thus, IE examines existing facts and rules and makes inferences by adding new knowledge when necessary. In addition, IE decides the order in which inferences are made.

In Protégé, reasoners are the inference engines capable of deriving new information from the existing ontology. For Protégé 5.2.0, several reasoners are available, such as ELK 0.4.3, HermiT 1.3.8.413, Ontop 1.18.1, Pellet, Pellet (Incremental), Jcel and Fact++. The reasoner for this study was Pellet, because it is more complete and capable to infer more complex relationships (HORRIDGE, 2011). Furthermore, Sirin et al. (2007) state that this reasoner has an optimal performance when used with the OWL language, allowing a more efficient inference in the presence of a large number of classes and individuals.

There are several methods for building ontologies, from those developed from scratch to the ones that use and adapt existing ontologies. Moreover, there are also several criteria and methods for evaluating ontologies, which may consider particular aspects of the model and its taxonomies (SERRA; GIRARDI; NOVAIS, 2014).

Several functionalities justify the construction of an ontology. One very important feature in the perspective of the Semantic Web is the possibility to respond to queries, since this allows users and applications to interact with the ontologies and data (KOLLIA; GLIMM; HORROCKS, 2011). In consonance with (STAAB; STUDER, 2009, p. 304), queries are competency questions that a specialist may want to submit to a domain's knowledge base in order to fulfill a given task.

Many search languages can be used in the context of ontologies. These languages can be classified into two categories: RDF-based search languages (e.g. RDQL, SeRQL and SPARQL), whose semantic language is based on the triple: subject, predicate, object; and search languages based on OWL DL (e.g. DIG ask queries and nRQL), which have a clear semantic language, but are not powerful enough in general cases (SIRIN; PARSIA, 2007). The SPARQL search language has an interface with OWL ontologies, allowing it to be more expressive and easily applied. In Protégé, the Snap-SPARQL plug-in is the framework for working with SPARQL and OWL (HORRIDGE; MUSEN, 2016).

The implementation of ontological models can potentially enrich the development process of new products, since manufacturing processes may benefit from previously unrecognized information for optimization purposes (i.e. unawareness). Thus, the implementation of ontologies in the context of organizations is associated with the SM principles, for all information becomes interconnected in order to create more reliable products and more efficient processes. These characteristics reflect the main concepts of the new industrial era, known as Industry 4.0, which is directly linked to the theme of this work.

\section{Methodological aspects}

A research project can be defined as a systematic investigation whose central theme is usually the development or refinement of theories and, in some cases, problem solving (GOUGH et al., 2012). The reasons that motivate one to conduct a research come from an identified gap in the theory or from some existing demand in practice. Hence, research with a more theoretical approach is generally known as pure research and its main objective is to achieve some abstract scientific progress. On the other hand, more practical research is known as applied research, whose main interest is the use of the results obtained to assist professionals in the solution of problems that occur in their daily work (SAUNDERS et al., 2012, p. 549). The present research project has a more practical bias, since it seeks to 
solve problems related to reliability, which is an important concern in the manufacturing industry.

One of the ways to characterize a research project is in relation to the proposed objectives of the study. Therefore, a descriptive research is intended to understand and describe certain phenomena. In research of this nature, real values are analyzed for the development of theories or hypotheses. Nevertheless, prescriptive research has the objective of recommending solutions and improvements to a problem, aiming to enhance the performance of the object of study. In this type of research, artifacts are created from data that are not necessarily true (HEVNER; CHATTERJEE, 2010; GIL, 2002). One may conclude by observing the objectives proposed by this present research that this work has a prescriptive nature, since its purpose is the development of a new artifact (a method) which may be used to improve existing processes.

Dresch et al. (2015) propose that the DSR approach should be adopted in the development of prescriptive research, because it is focused on problem solving. Furthermore, Hevner and Chatterjee (2010, p. 5) argue that the fundamental principle of DSR is that the understanding of a design problem and its solution should be used in the construction and application of an artifact. Peffers et al. (2007) propose a flowchart to conduct a DSR-based research, as shown in Figure 1.

\section{Development of the method}

In this section, the obtained results are presented, following the steps of the DSR approach as described in the methodological aspects.

\subsection{Problem identification and definition of expected results}

In order to identify the central problem of this research, a bibliometric review was carried out on recent papers related to NPD, DfR and product failures. From this activity, some research opportunities were encountered. No reports of the application of ontological models for DfR implementation in the initial stages of the NPD were observed in the bibliographic portfolio.
Interviews and meetings with professionals responsible for the Product Engineering and Manufacturing Engineering departments at a global tractor manufacturing company located in Brazil were important to identify a practical research opportunity. It was verified that the starter motors belonging to a new tractor model are a critical component in the tractor's performance due to some detected failures when the equipment starts its operation in the field. Although the company has a specification manual for the testing plan that suppliers are required to follow, the equipment has continued to fail during its operation. Thus, one may conclude that the adopted test plan for those starter motors might not be appropriate according to its field usage profile. This fact is likely to happen due to the reuse of projects (and reuse of test plans), which often occurs during the elaboration of new products. Therefore, those existing projects might not be suitable to the specific needs of the new product.

The company provided some official documents to assist in the problem analysis and in the elaboration of the solution. Manuals related to the investigated item's development process and a guidebook with technical specifications allowed a better understanding of its use and manufacture. In addition, a compendium containing all test specifications to which mechanical and electronic components must be submitted was used as supplementary material. All these documents were considered in the study to find some possible inconsistencies that would support the development of the proposed solution.

Manuals related to the investigated item's development process and a guidebook with technical specifications allowed a better understanding of its use and manufacture. In addition, a compendium containing all test specifications to which mechanical and electronic components must be submitted was used as supplementary material. All these documents were considered in the study to find some possible inconsistencies that would support the development of the proposed solution. From the analysis of the materials supplied by the company and the information provided by the engineers, it was possible to conclude that the tests

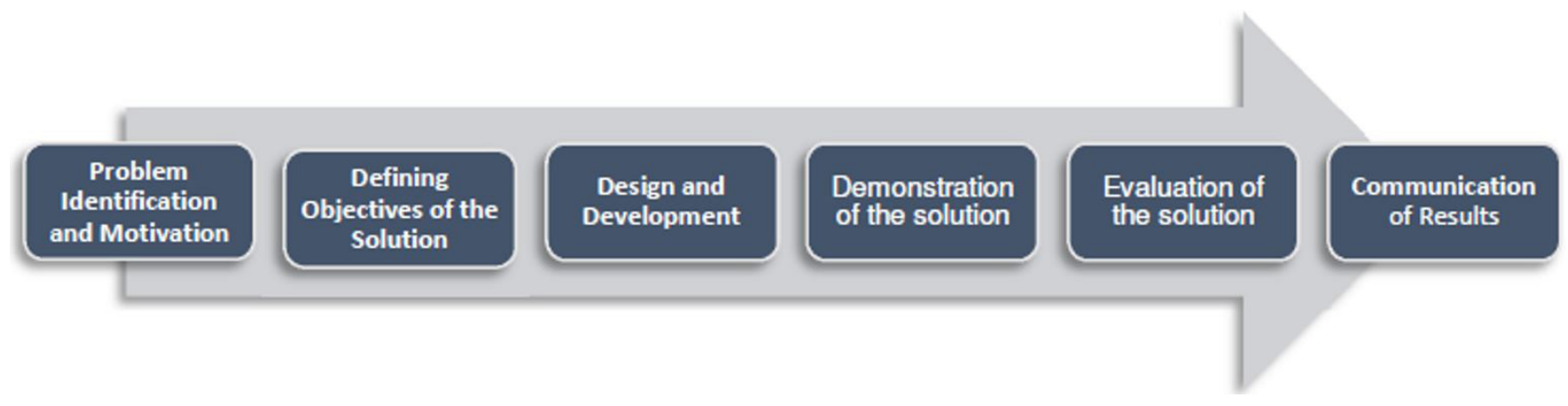

Figure 1. Method to implement the DSR approach. Source: Peffers et al. (2007). 
carried out on those starter motors do not consider their interaction with the environment and the specific product's conditions of use. Moreover, the performed tests are oriented to products developed to operate in a specific location and this does not ensure that the product will present the same reliability if used in an adverse condition. Finally, it was possible to observe that some tests that could be waived are executed, whilst other relevant tests are not given proper consideration.

\subsection{Ontological model development}

The method to construct the ontological model adopted in this research was Method 101. A summary of the main steps for the development of an ontology following this method can be visualized in Figure 2. The process of creation an ontological model following this method will be presented as follows.

\subsubsection{Determination of domain and scope of the ontology}

From the analysis of the information and documents provided by the company, some inconsistencies in the test plan of starter motors of a certain type of tractors were verified. Considering this information, the scope of this research was defined. It was stipulated that the created ontology should be able to support the NPD team in the test planning of starter motors, according to the external conditions and the usage profile of the tractor. It is expected an improvement in the reliability of this equipment.

Two hypothetical scenarios of usage were created. These scenarios were fundamental for the delimitation of the competency questions related to the ontological model. Thus, in order to demonstrate and evaluate the proposed model, it was necessary to analyze both scenarios and answer the questions.

According to the manuals provided by the company, starter motors, which are responsible for driving the tractors' motor, are located in a protected position inside the vehicle. Even so, these items can be subjected to elements present in the external location, such as organic matter, rain, snow, dust, high temperatures and chemicals. In agreement with the electromechanical requirements manual, the presence of those elements may compromise the proper functioning of such equipment.

In view of this information, it was defined that, among the various tests that the company is able to perform, the tests related to high temperature, dust ingress, chemical resistance and humidity were selected for analysis by the proposed ontology. From this perspective, the features of two hypothetical scenarios, which could have some influence on the tractors and the tests, were also defined.

The first scenario is related to the use of tractors in sugarcane crops in the central region of the state of São Paulo. This is one of the most relevant sugarcane producing regions in Brazil. The second scenario is related to the use of tractors in soybean plantations. The represented region is the west of Paraná state, one of the main producers of this grain in this South-American country. After selecting these two scenarios, a characterization of these locations was carried out according to the physical and geographical aspects of each region. Such information is described in Table 1.

Thus, by analyzing the information about the starter motors and the selected scenarios, competency questions that support the development of this ontological model were defined:

- What is the most recommended starter motor to be used in a given scenario, observing the reliability tests to which this equipment is subjected?

- What reliability tests should be performed on a starter motor that will operate on the crop of a specific product?

- What is the best test procedure to perform in a product in order to ensure its reliability?

A search for existing ontology models for the test planning of a starter was performed in online libraries

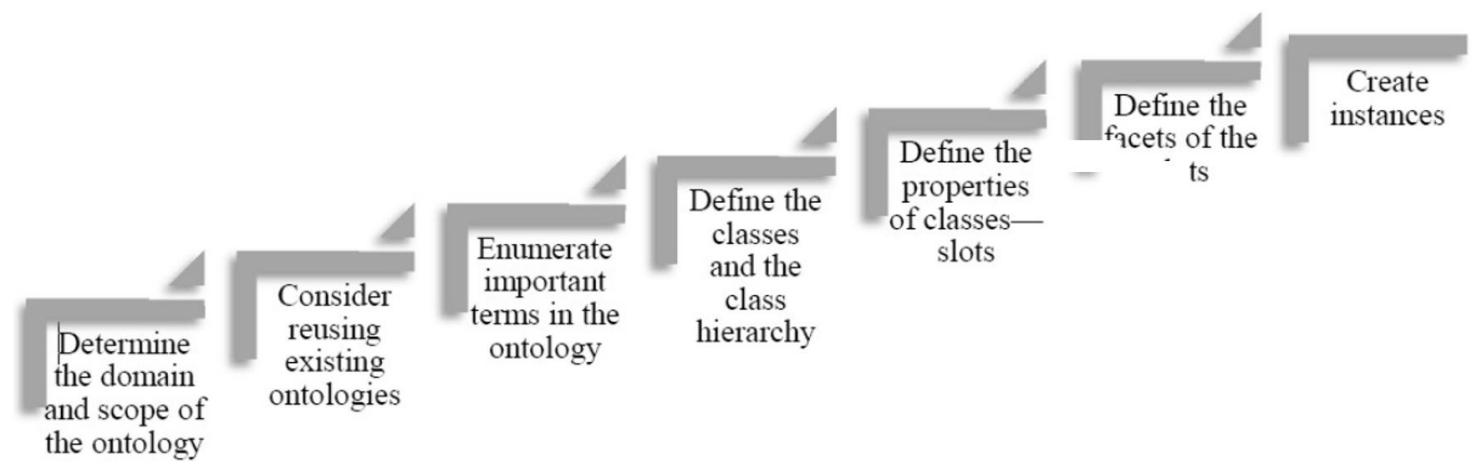

Figure 2. Summary of main activities performed through the Method 101. Source: Adapted from Noy and MacGuinness (2001). 
Table 1. Hypothetical scenarios used for the ontology creation process.

\begin{tabular}{|c|c|c|}
\hline DESCRIPTION & SCENARIO 1 & SCENARIO 2 \\
\hline Location & São Paulo state - Brazil & Soybeans \\
\hline Final Product & Sugarcane & $30^{\circ} \mathrm{C}$ \\
\hline Maximum Temperature & $40^{\circ} \mathrm{C}$ & $20^{\circ} \mathrm{C}$ \\
\hline Average Temperature & $25^{\circ} \mathrm{C}$ & $7{ }^{\circ} \mathrm{C}$ \\
\hline Minimum Temperature & $19^{\circ} \mathrm{C}$ & $150 \mathrm{~mm}$ \\
\hline Average Precipitation & $121 \mathrm{~mm}$ & $45 \%$ \\
\hline Humidity Ratio & $75 \%$ & Red Latosol (dryer) \\
\hline Soil Type & Purple Latosol (more humid) & $4.80 \mathrm{kWh} / \mathrm{m}^{2}$ \\
\hline
\end{tabular}

Source: The authors.

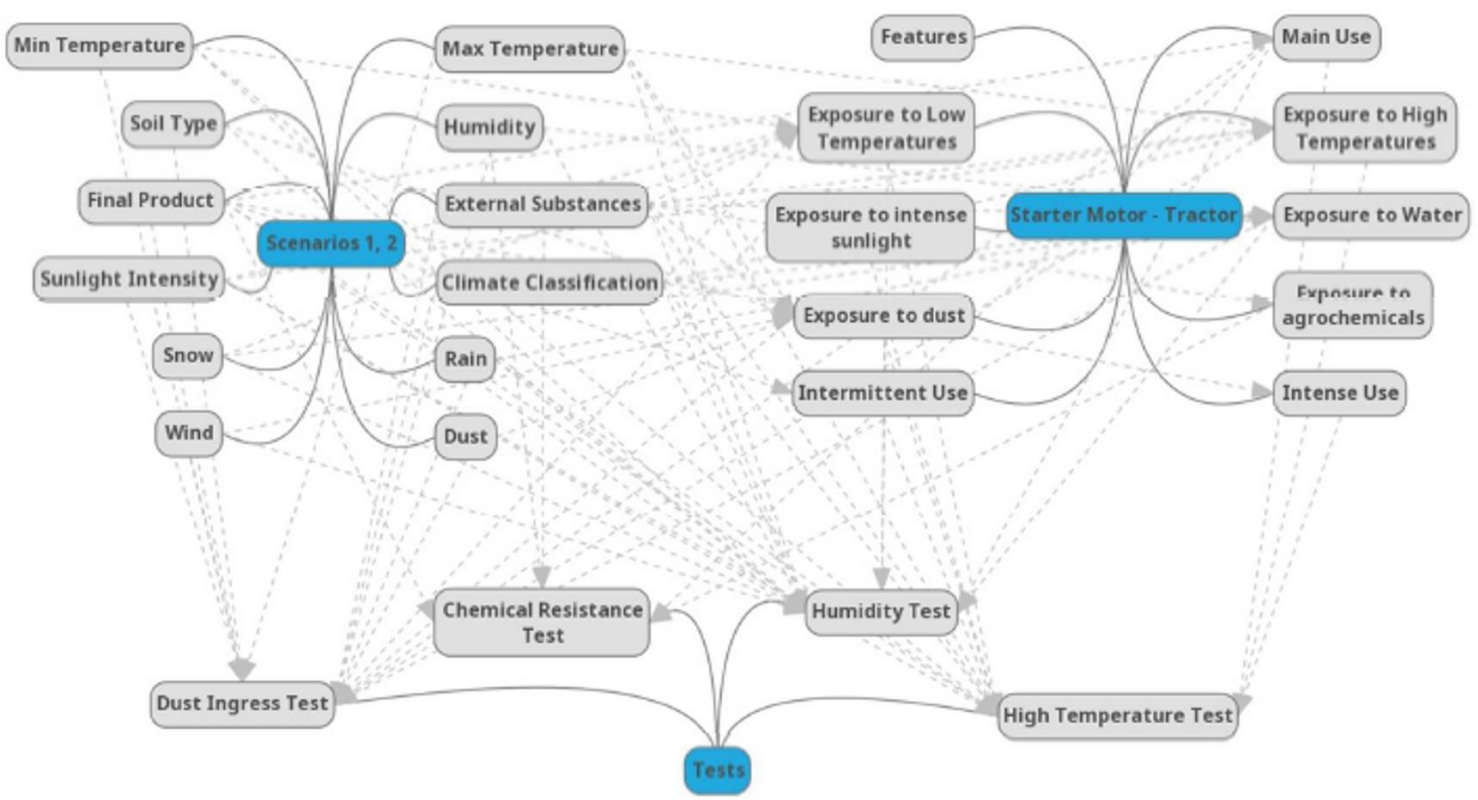

Figure 3. Mind map of concepts related to the domain of ontology. Source: The authors.

(DMOZ, 2019; DAML ONTOLOGY LIBRARY, 2019). Because this is a specific ontology developed for a particular purpose, there are no similar existing models. Hence, the second step of method 101, i.e. "considering reusing existing ontologies", could not be satisfied. Thus, the development of a model starting from scratch was performed. The next stage for the construction of the ontological model refers to the enumeration of important terms to be adopted in the ontology.

\subsubsection{Enumeration of important terms for this ontology}

Given that an ontology is a representation of terms and taxonomies, the enumeration of concepts in the model is important to verify some existing possible relations and classifications among the elements. A mind map with words and concepts related to the ontology domain was created. The knowledge acquired from the relationships among the individuals in the list allows the creation of ontology entities, such as classes, properties and individuals. According to Figure 3 it is possible to perceive a representation of ideas and concepts present in the developed ontological model.

\subsubsection{Defining classes and hierarchies}

After the enumeration of terms and relations within the domain proposed for the ontological model in this work, the process of defining the classes and hierarchies began. The top-down development process, which starts from the determination of more generic classes to the most specific ones, was adopted. Hence, the ontology editor Protégé 5.2.0 was used to define the taxonomies of the model. The following classes were created: Features, Scenarios, StarterMotorTractor, StarterMotorTractorInteraction and Tests. The hierarchy of classes and subclasses of the model as a representation of the taxonomy can be seen in Figure 4.

Class Features contains all the attributes related to the scenarios where the starter motors are designed to operate. 
Thus, some information related to the environment and the final product cultivation are described in this class. In class Scenarios, the tractors' scenarios of use are described. Moreover, two hypothetical scenarios of use were created in the form of individuals. Class StarterMotorTractor presents two starter motors that were created in the form of individuals. These items operate in different scenarios, and, therefore, behave following their different interactions with the location. Such interactions are described in class StarterMotorTractorInteraction, in which some information related to the tractor's interaction with the scenario and usage profile are represented. Finally, class Tests contains the tests that the starter motors undergo according to their

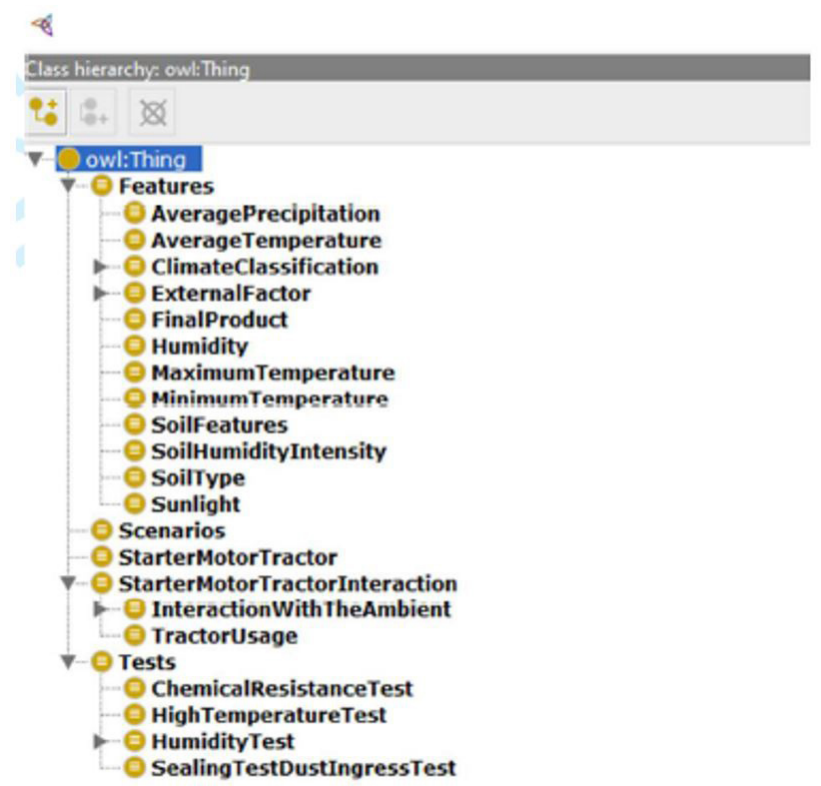

Figure 4. Class taxonomy of the proposed ontology model in the editor Protégé 5.2.0 (STANFORD CENTER FOR BIOMEDICAL INFORMATICS RESEARCH, 2017). Source: The authors. usage profile and interactions with the location in which they are described to be working.

\subsubsection{Defining class properties and characteristics and creating instances}

From the hierarchy of classes and subclasses presented in section 4.2.3, it was necessary to define the object properties as well as the data properties. Object properties are important for relating objects (class instances or domain elements) to other objects (STAAB; STUDER, 2009, p. 97). An example of object property can be found in Figure 5. In this example, the property exhibited is hasTest, which possess class StarterMotorTractor as a domain (since it contains the instances that receive the tests) and class Tests as a range (once that all available tests are inserted in this class).

In addition, data properties are important for relating objects to values of data types (STAAB; STUDER, 2009, p. 97). Figure 6 illustrates data property hasMaximumTemperatureValue. In this example, the range was determined as double, which is a data type that represents decimal values. For the case of this property, it is also classified as Functional, since each object can receive only a unique maximum temperature value.

Through the development of the model, several changes have occurred regarding the taxonomy, properties and individuals aiming to a better representation of the research domain. In order to check the inconsistencies and to make inferences in Protégé 5.2.0, a reasoner was used. According to Staab and Studer (2009, p. 107), reasoners should be used to check the consistency of an ontology in OWL, i.e., search the presence of any logical contradictions in the axioms of the model. Moreover, reasoners may derive inferences from all the information introduced in the model, i.e. infer whether a particular individual on an ontology belongs to a specific class.

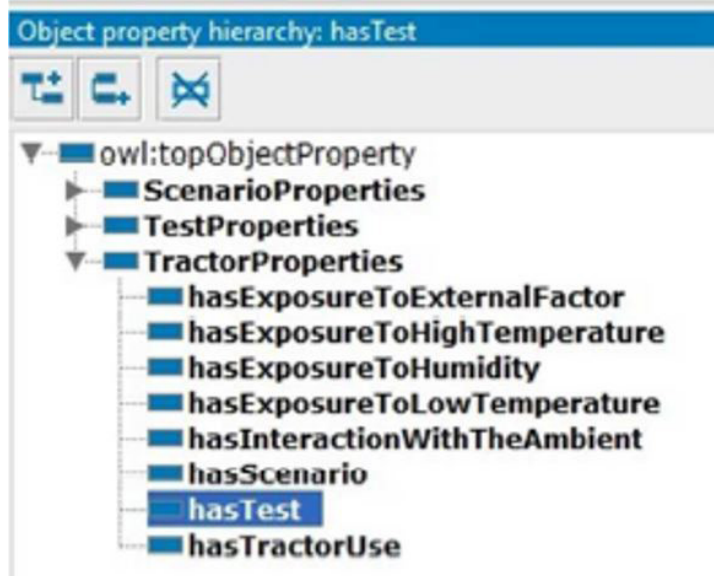

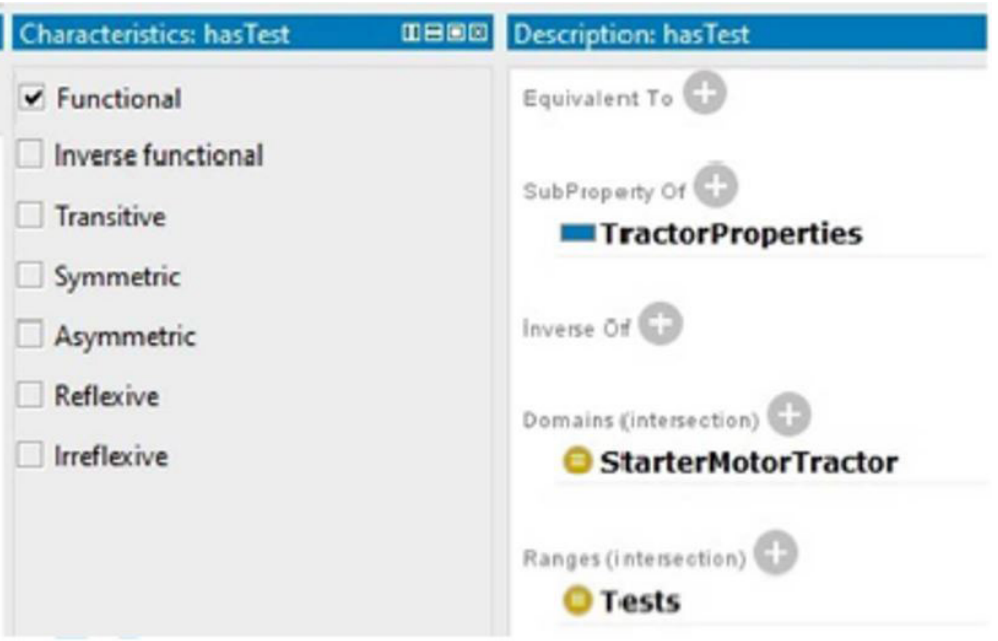

Figure 5. Object Property Example - Has test. Source: The authors. 


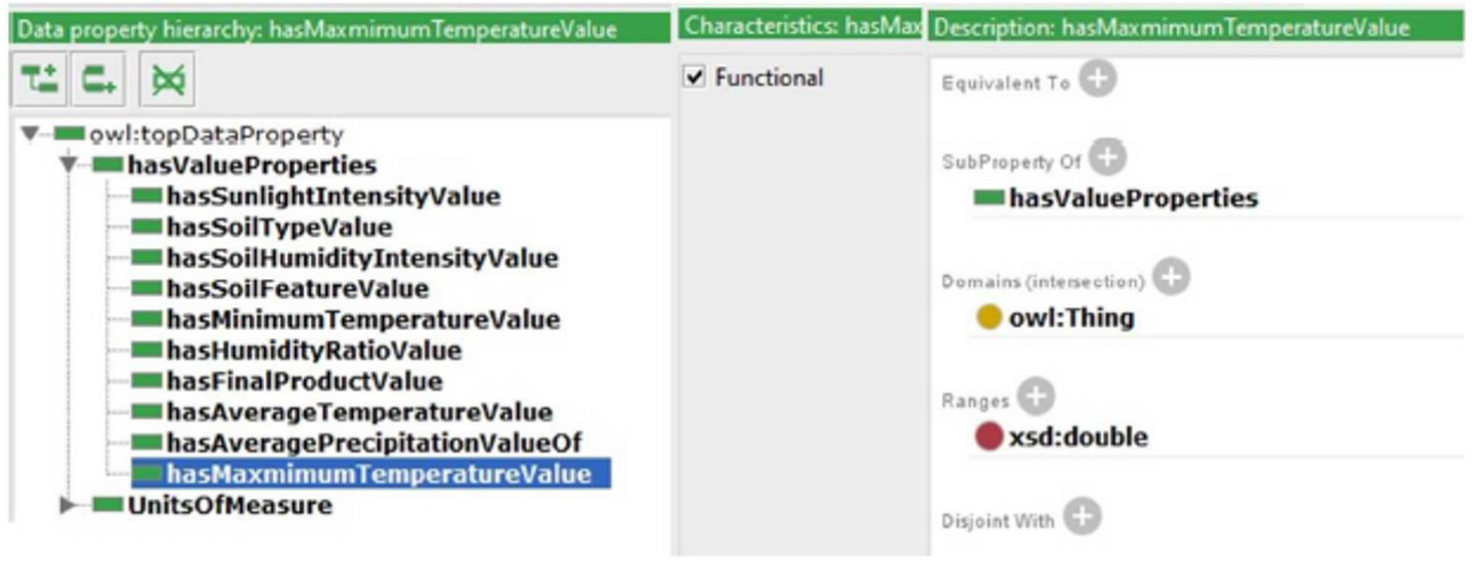

Figure 6. Data property example - has Maximum Temperature Value. Source: The authors.

Thus, the Pellet reasoner was used through the ontology construction process to verify inconsistencies and to perform inferences in the model. In addition, the tool "Debug Ontology..." was used to identify any incoherence for later correction. At the end of the model construction, no error was found and the model was able to perform logical inferences. The importance of the reasoner to realize inferences and establish connections among individuals or classes makes ontological models useful tools for a peculiar domain (AHLERS et al., 2015).

With the conclusion of the steps proposed by Method 101, the ontological model operates effectively and is able to answer the previously specified competency questions. The following section corresponds to the fourth step of the DSR framework, which is based on the demonstration of the proposed solution. In the case of the constructed ontology in this research, some queries were elaborated with the objective of verifying whether the model is able to answer the defined competency questions.

\subsection{Demonstration of the proposed solution}

The demonstration of the solution proposed in this research was accomplished by performing some queries on the ontology. The Snap-SPARQL plug-in was used in Protégé 5.2.0 to execute this activity. This plug-in was chosen because, according to Horridge and Musen (2016), it presents several tools and functions that help users in the construction of queries. In addition, Snap-SPARQL can be effectively used for OWL ontologies by interacting with all Protégé 5.2.0 reasoners. In this way, queries were developed in the SPARQL language in order gather the desired information.

In agreement with Grüninger et al. (1995), the development of ontologies is motivated by applications they may have in different scenarios, which are usually presented in the form of problems or examples not solved by existing ontologies. Hence, the use of scenarios makes the understanding of the motivation and the application of a created model feasible.

The queries performed on this research pursued to identify whether the developed ontology would be able to properly respond to some previously established information and inferences. As reported by Grüninger et al. (1995), queries can be considered requirements in the form of questions that an appropriate ontology must be able to answer. The implementation of queries on an ontology provides an initial assessment of a model, since it enables the user to determine whether competency questions are able to be fulfilled.

According to Staab and Studer (2009, p. 502), a query is composed of two components, SELECT and WHERE. The first part is responsible for specifying the projection, i.e., the variables that must be extracted as a result of the searches. The second component corresponds to the constraints that must be present in the possible search solutions.

The response to the first established competency question and its correspondent query is presented in Figure 7. As described in the section 4.2.1, the first competency question strives for the identification of the most recommended starter motor to be used in each scenario, observing the reliability tests to which this equipment is subjected. As Figure 7 depicts, one can realize the identification of the most appropriate starter motor to operate in each scenario. Thus, looking at the results of the query, it is possible to state that for Scenario 1 (i.e., Scenario__Sugar Cane Sao Paulo) the starter motor 1 (i.e., StarterMotorTractorl) is the most recommended to operate whereas for scenario 2 (i.e., Scenario2_Soy Beans Parana) the starter motor 2 (i.e., Starter Motor Tractor2) is the most indicated. 


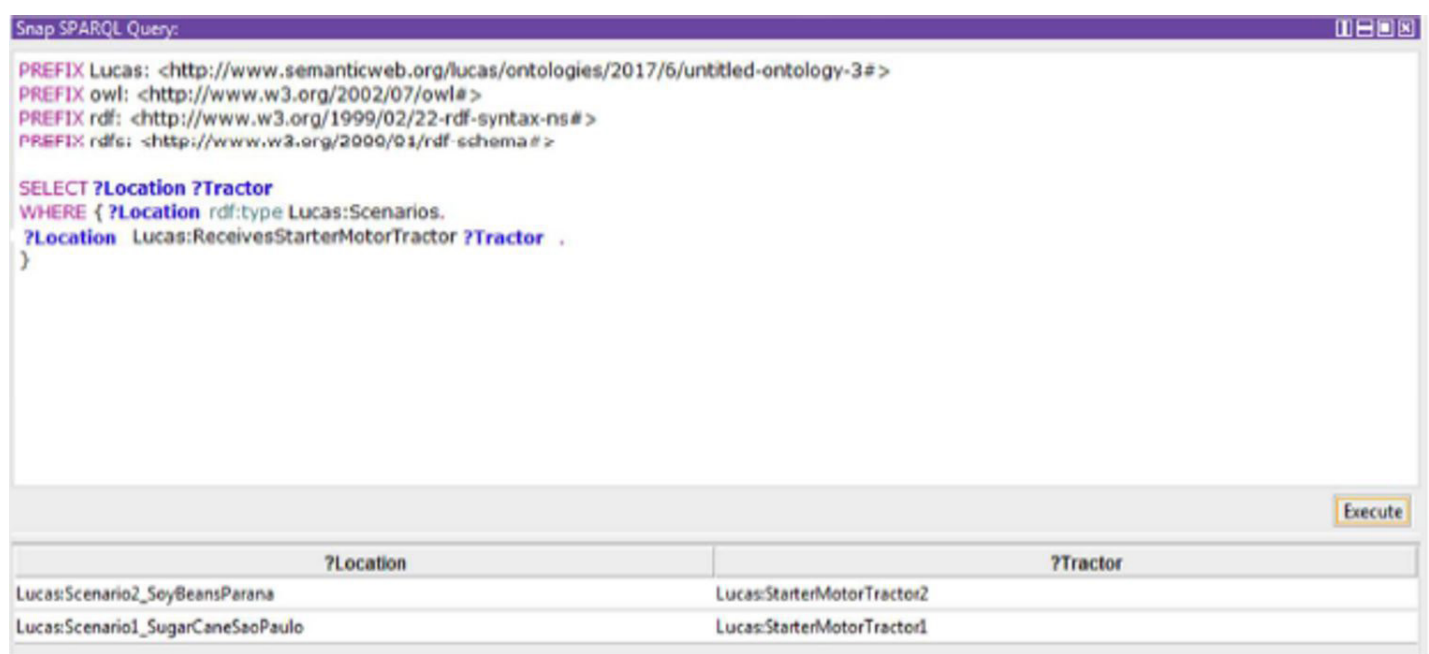

Figure 7. Identification of the indicated starter motor for each scenario. Source: The authors.

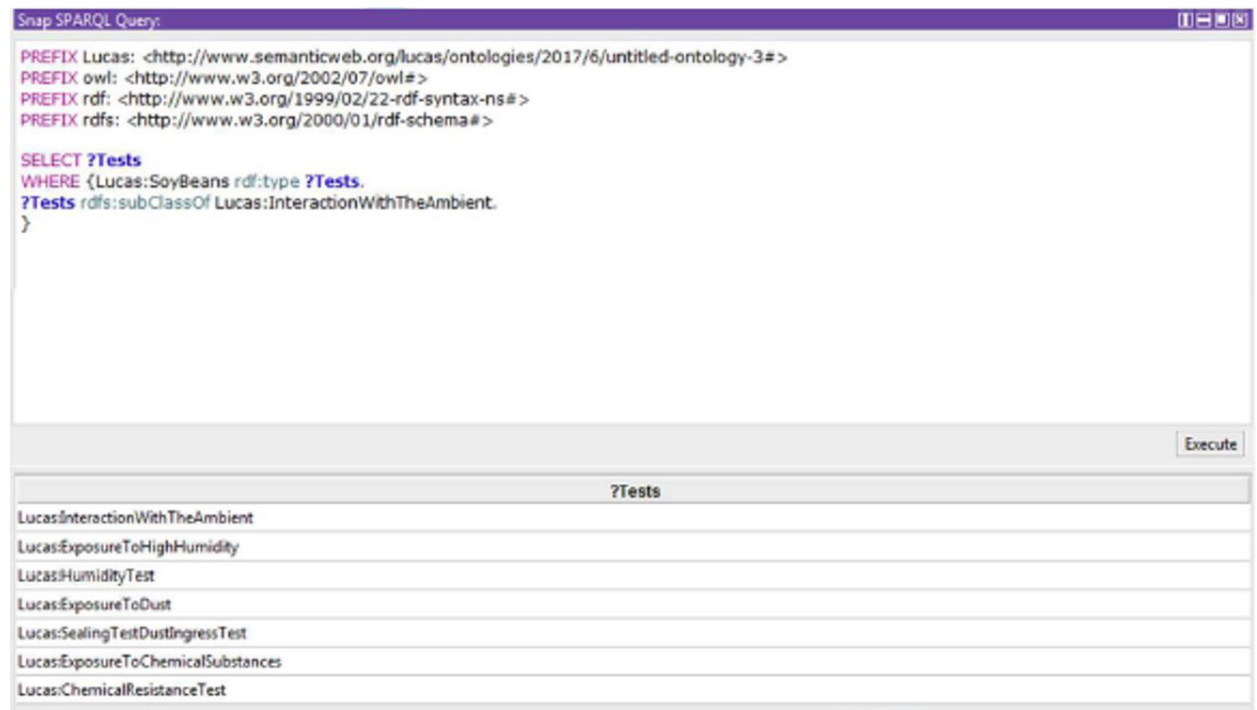

Figure 8. Identification of the interaction and tests that a tractor has according to a field crop. Source: The authors.

The following query has the purpose of answering the second competency question: What reliability tests should be performed on a starter motor that will operate on the crop of a specific product? This query aims to assist the NPD engineering team in identifying the main reliability tests that must be performed on a starter motor that will be used in a determined crop. Thus, when establishing the product/scenario to which the product will be developed, engineers will be able to identify all the mandatory tests that must be performed to ensure the equipment's reliability. These tests are determined in line with the interactions that the starter motor has with the plantation ambient and the conditions of use to which they will be manipulated. The result of this query is represented in Figure 8, which shows the interactions that the equipment has with the environment and the respective tests.
The last query pursues to represent test procedures. Furthermore, it represents the last competency question described in chapter 4.2.1: What is the best test procedure to perform in a product in order to ensure its reliability. In the previous example (Figure 8), it is possible to perceive that starter motors operating on soybean crops (i.e., SoyBeans) should undergo the humidity test (i.e., HumidityTest), since the equipment may be exposed to high humidity in these environments. Thus, the procedure for this test varies according to the intensity of humidity that the equipment is exposed. Locations are analyzed and classified in type 1, type 2 or type 3 according to their humidity ratio and external substances because in each situation the test procedures should vary. The classification of the scenario related to soybean crops according to its humidity ratio is represented in Figure 9. Moreover, the test procedures to 


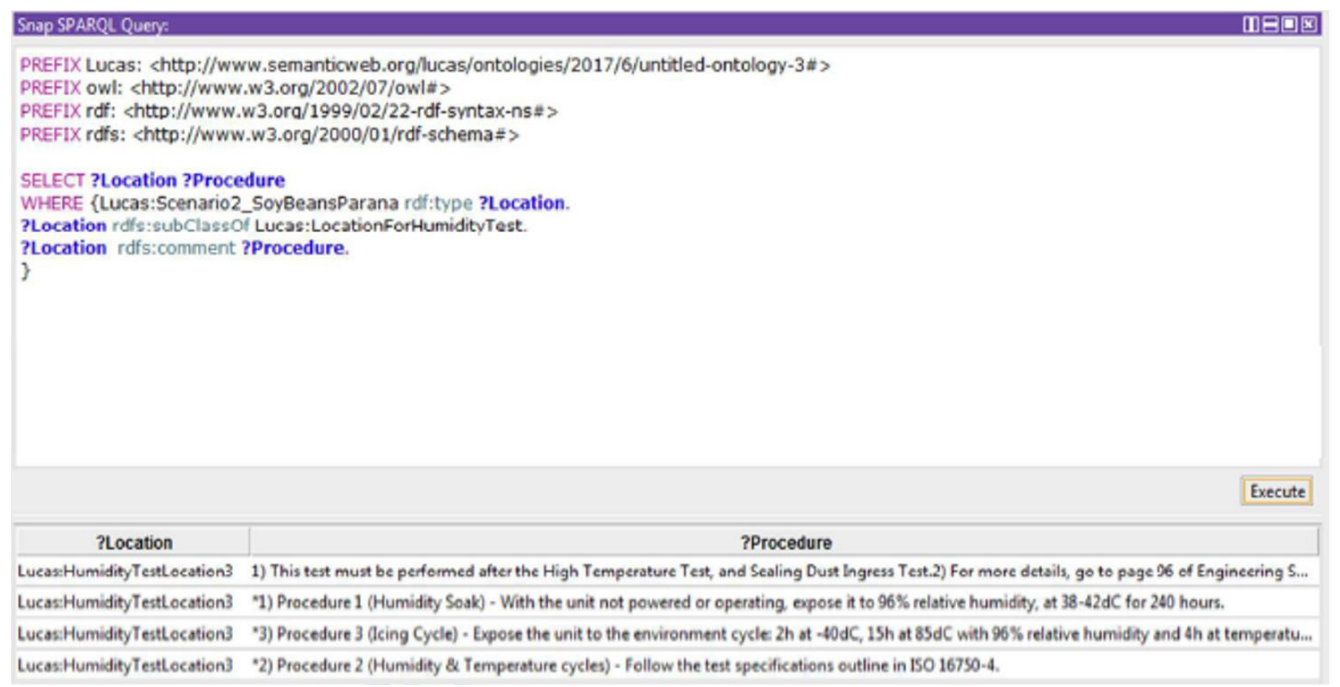

Figure 9. Identification of the type of site and respective procedures for humidity test. Source: The authors.

be performed on an equipment operating in this scenario are also demonstrated.

\subsection{Evaluation of the developed artifact}

The fifth step of the DSR framework consists of evaluating the model. The researcher should observe and evaluate the behavior of the artifact in the problem solution. A comparative analysis was carried out within the expected objectives, to verify to what extent the previously defined expectations were met. Following some predetermined criteria, such as the ease, feasibility, quality and effectiveness, the ontology was analyzed.

Fernandez-Breis et al. (2009) state that the proposal to evaluate an ontology must consider different perspectives, which can be grouped according to their objectives. The first group corresponds to the evaluation according to structural properties of the ontology, that is, according to classifications and accuracy of the model. On the other hand, the second group is associated with characteristics related to quality, e.g. usability, reliability and functionality. Furthermore, these same authors affirm that the development process of an ontology contains characteristics compared to Software Engineering, and, therefore, its evaluation must occur according to this same perspective, using the (INTERNATIONAL ORGANIZATION FOR STANDARDIZATION, 2000).

Following the concepts of the ISO 9126 (INTERNATIONAL ORGANIZATION FOR STANDARDIZATION, 2000), which represents an international standard for the evaluation of software quality, the assessment of an ontology should consider the internal, external and in use aspects of the model. Internal aspects are those referring to the ontology itself and should be evaluated during its development process. Nevertheless,

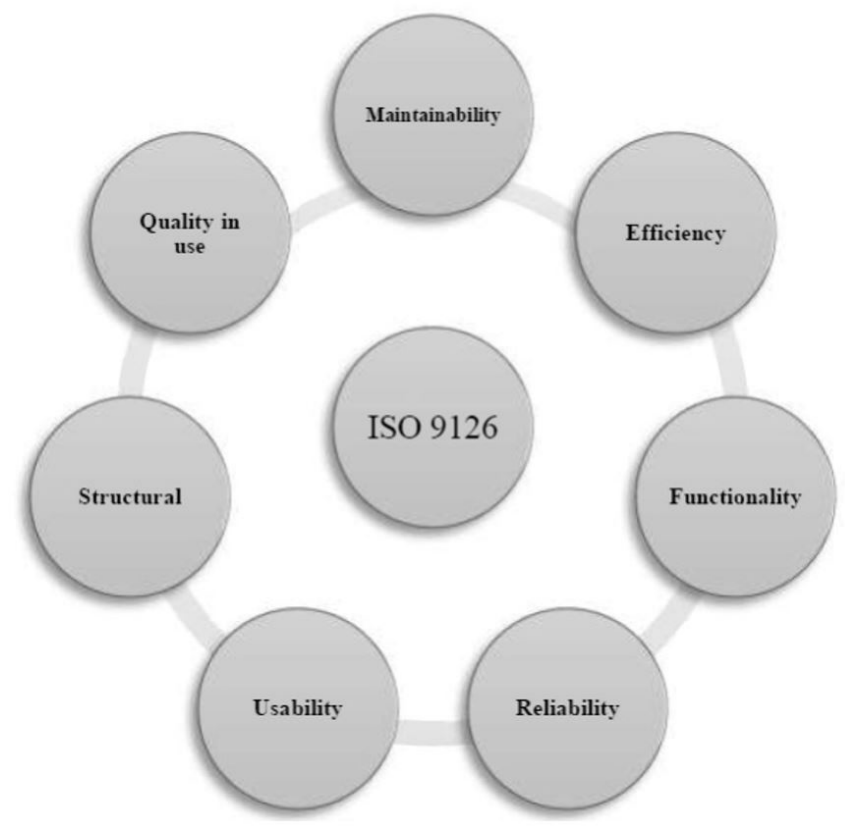

Figure 10. Dimensions for ontology evaluation based on the ISO 9126 (INTERNATIONAL..., 2000). Source: FernandezBreis et al. (2009).

external aspects are related to the behavior of the model and must be evaluated in the final stages of development or in the testing stages, before the ontology release. The in-use aspects should be evaluated after the ontology is launched in order to make corrections on possible errors. Observing these aspects, Fernandez-Breis et al. (2009) propose that the evaluation structure of an ontology should comprise seven dimensions described in Figure 10.

In order to accomplish this stage, the presence of the developer of this ontology together with a professional 
Table 2. Evaluation criteria in each of the dimensions.

\begin{tabular}{|c|l|l|}
\hline Structural & \multicolumn{1}{|c|}{ Criteria } & Overall Evaluation \\
\hline Dimension for Analysis & $\begin{array}{l}\text { - Accuracy } \\
\text { - Cohesion } \\
\text { - Consistency } \\
\text { - Integrality }\end{array}$ & Achieves the goal \\
\hline Functionality & $\begin{array}{l}\text { - Inferencing } \\
\text { - Results representation } \\
\text { - Classifying instances } \\
\text { - Consistent search and query }\end{array}$ & Achieves the goal \\
\hline Reliability & $\begin{array}{l}\text { - Maturity } \\
\text { - Robustness }\end{array}$ & Achieves the goal \\
\hline Usability & $\begin{array}{l}\text { - Changeability } \\
\text { - Testability }\end{array}$ & Achieves the goal \\
\hline Maintainability & $\begin{array}{l}\text { - Changeability } \\
\text { - Testability }\end{array}$ & Achieves the goal \\
\hline Quality in use & $\begin{array}{l}\text { - Effectiveness } \\
\text { - User satisfaction }\end{array}$ & Achieves the goal \\
\hline Efficiency & - Computational efficiency & Achieves the goal \\
\hline
\end{tabular}

Source: ISO 9126 ( INTERNATIONAL ORGANIZATION FOR STANDARDIZATION, 2000), Fernandez-Breis et al. (2009). The authors.

responsible for the Product Engineering department from the partner company was necessary. The purpose of this stage was to verify if the created ontology was convenient and representative for the research domain.

According to the ISO 9126 ( INTERNATIONAL ORGANIZATION FOR STANDARDIZATION, 2000), the evaluation scale must follow some requirement metrics of satisfaction. For example, the evaluation scale can be divided into two categories: satisfactory or unsatisfactory; or even into four categories: exceeds expectations, achieves the goal, minimally acceptable and unacceptable. For the evaluation of this ontology, the evaluation scale divided into four categories was adopted to facilitate the analysis of the items likely to be improved.

Therefore, some of the criteria were evaluated only by the developer, whereas others were evaluated together with a professional from the partner company. It was established that the dimensions Functionality, Reliability, Maintenance and Efficiency had to be verified by the developer of the model since they are related to the internal and external aspects of the ontology. On the other hand, the Structural, Usability and Quality in use dimensions were evaluated together with a representative of the company, since these items are linked to the usage aspects of the model and require the analysis of a specialist about the domain. Thus, the concepts evaluated in each dimension and the analyzed criteria are represented in Table 2.

\section{Final considerations}

The results obtained from this work reveal that it is possible to perceive the importance of understanding the existing interactions among external factors, usage profile and reliability of products. Firstly, this fact becomes relevant to establish the most appropriate test plan of an item according to the location and conditions of use in which it will operate. In addition, the identification of the product's usage profile can assist engineers in the best choice of materials, parts and design that will ensure the reliability of a system as a whole. Therefore, the implementation of DfR-oriented activities in the early stages of the NPD can result in significant improvements in the quality and reliability of products. This brings several benefits to the company, which avoids corrective expenses or inconveniences related to the brand's reputation.

The development of this ontological model occurred in the Protégé 5.2.0 editor. From the data modeling, it was possible to observe how certain factors can directly influence the reliability and the test planning of a product. One of the major limitations that arose in the development of this ontology was related to Protégé 5.2.0. The software in many situations is slow and, in the presence of some inconsistency within the taxonomy, it cannot accurately indicate the error. Thus, in order to solve a problem of this nature, one can take a long time to fix the error.

The use of the DSR framework in this research project was important for delimiting the steps to be followed to accomplish the method. Furthermore, this methodology approach clearly indicates how to perform each stage of work, in an attempt to create an artifact that can solve some problem. This fact makes the DSR approach recommended for prescriptive research such as this one.

The scientific contribution of this work is based on the fact that it is an innovative prescriptive research that involves the use of ontologies to assist the implementation of DfR in the initial stages of the NPD. Looking at the most recent studies on the subject, it was not observed any 
research on this nature. Thus, the developed method has the purpose of assisting NPD teams with the creation of more reliable and resistant products in accordance with the conditions of use to which they will be handled. The results were presented to the partner company and it was stated that this method could be implemented in the initial stages of the NPD. Hence, it is expected that the determination of a more effective test plan for starter motors will improve their reliability.

The creation of a simpler platform to the end user, which should have some interface with the OWL language, emerges as a future recommendation for this work. This platform should allow the user to search and include data and relationships without requiring much knowledge about OWL languages or ontological modelling. Moreover, it is recommended that the proposed method should be expanded by adding some real usage scenarios, as well as it should be revaluated by including more tests and specifications.

Finally, although the proposed method requires a team of specialists who have some knowledge on ontologies to be properly used, the developed model was able to solve an existing problem in the company's reality. In this way, it proves to be feasible for implementation not only in the context of starter motors but also in other items.

\section{References}

AHLERS, D. et al. Challenges for information access in multi-disciplinary product design and engineering settings. In: INTERNATIONAL CONFERENCE ON DIGITAL INFORMATION MANAGEMENT, 10., 2015, USA. Proceedings... USA: IEEE, 2015. p. 109-114. http://dx.doi. org/10.1109/ICDIM.2015.7381865.

AKERKAR, R.; SAJJA, P. Knowledge-based systems. Sudbury: Jones \& Bartlett Publishers, 2010.

ASHRAFI, M.; DAVOUDPOUR, H. A hierarchical bayesian network to compare maintenance strategies based on cost and reliability. International Journal of Industrial Engineering: Theory Applications and Practice, v. 26, n. 3, p. 1-11, 2019.

BILGIN, G.; DIKMEN, I.; TALATBIRGONUL, M. Ontology evaluation: an example of delay analysis. Procedia Engineering, v. 85, p. 61-68, 2014.

BOUNCKEN, R. B. et al. Coopetition in new product development alliances: advantages and tensions for incremental and radical innovation. British Journal of Management, v. 29, n. 3, p. 391-410, 2018. http://dx.doi. org/10.1111/1467-8551.12213.

BRALLA, J. G. Design for excellence. New York: McGrawHill, 1996.

CORCHO, Ó. et al. ODEval: a tool for evaluating RDF(S), DAML+OIL, and OWL concept taxonomies. In: M Bramer, M.; Devedic, V. Artificial intelligence applications and innovations. Boston: Kluwer Academic Publishers, 2004. p. 369-382. http://dx.doi.org/10.1007/1-4020-8151-0_32.

CROWE, D.; FEINBERG, A. Design for reliability. Boca Raton: Press CRC, 2001.

CUI, A. S.; WU, F. The impact of customer involvement on new product development: contingent and substitutive effects. Journal of Product Innovation Management, v. 34, n. 1, p. 60-80, 2017. http://dx.doi.org/10.1111/jpim.12326.

DA XU, L.; XU, E. L.; LI, L. Industry 4.0: state of the art and future trends. International Journal of Production Research, v. 56, n. 8, p. 2941-2962, 2018. http://dx.doi.or g/10.1080/00207543.2018.1444806.

DAML Ontology Library. Available from: $<$ http://www.daml. org/ontologies/>. Access in: 13 Dec. 2019.

DMOZ. Available from: <http://www.dmoz.org/>. Access in: 13 Dec. 2019.

DRESCH, A.; LACERDA, D. P.; ANTUNES JUNIOR, J. A. V. Design science research: a method for science and technology advancement. New York: Springer, 2015.

FERNANDEZ-BREIS, J. T. et al. Quality evaluation framework for bio-ontologies. In: INTERNATIONAL CONFERENCE ON BIOMEDICAL ONTOLOGY, 2009, Buffalo. Proceedings... Manchester: Nature Proceding, 2009. p. 127-131.

GANGEMI, A. Ontology design patterns for semantic web content. In: Lecture notes in computer science: including subseries lecture notes in artificial intelligence and lecture notes in bioinformatics. In: INTERNATIONAL SEMANTIC WEB CONFERENCE, 4., 2005. Proceedings... Galway, Ireland: Lecture Notes in Computer Science, vol. 3729. p. 262-276. http://dx.doi.org/10.1007/11574620_21.

GENNARI, J. H. et al. The evolution of Protégé: an environment for knowledge-based systems development', International Journal of Human-Computer Studies, v. 58, n. 1, p. 89-123, 2003. http://dx.doi.org/10.1016/S10715819(02)00127-1.

GIL, A. C. Como elaborar projetos de pesquisa: métodos e técnicas de pesquisa social. 6. ed. São Paulo: Atlas, 2002. p. 22-23.

GOUGH, D. et al. An introduction to systematic reviews. London: Sage Publications Ltd., 2012.

GRUBER, T. R. A translation approach to portable ontology specifications. Knowledge Acquisition, v. 5, n. 2, p. 199220, 1993. http://dx.doi.org/10.1006/knac.1993.1008.

GRÜNINGER, M.; FOX, M. S.; GRUNINGER, M. Methodology for the design and evaluation of ontologies. In: INTERNATIONAL JOINT CONFERENCE ON ARTIFICIAL INTELIGENCE (IJCAI95). WORKSHOP ON BASIC ONTOLOGICAL ISSUES IN KNOWLEDGE SHARING, 1995, Galway. Proceedings... Galway, Ireland: Computer Science . p. 1-10. 
HEVNER, A.; CHATTERJEE, S. Design research in information systems: theory and practice. USA: Springer Science \& Business Media, 2010. v. 22.

HORRIDGE, M. Justification based explanation in ontologies. 2011. 304 f. Thesis (PhD in Computer Science). Engineering and Physical Sciences, University of Manchester, Manchester, Inglaterra, 2011.

HORRIDGE, M.; MUSEN, M. Snap-SPARQL: a java framework for working with SPARQL and OWL. In: Ünay, D. Çataltepe, Z. Aksoy, S. Lecture notes in computer science: including subseries lecture notes in artificial intelligence and lecture notes in bioinformatics. USA: Springer Verlag, 2016. p. 154-165. http://dx.doi. org/10.1007/978-3-319-33245-1_16.

INTERNATIONAL ORGANIZATION FOR STANDARDIZATION - ISO. ISO 9126: Information technology-software product quality. Geneva: ISO, 2000.p. 34.

KOLLIA, I.; GLIMM, B.; HORROCKS, I. SPARQL query answering over OWL ontologies. In: Crawford, B. Castro, C. Monfroy E.. Lecture notes in computer science: including subseries lecture notes in artificial intelligence and lecture notes in bioinformatics. USA: Springer Verlag, 2011. p. 382-396. http://dx.doi.org/10.1007/978-3-642-21034-1_26.

KUEI-CHEN, C.; YEU-SHIANG, H.; I-CHI, H. A study of software reliability growth with imperfect debugging for time-dependent potential errors. Computer-Aided Design, v. 26, n. 3, p. 376-393, 2019. http://dx.doi.org/10.15675/ gepros.v12i4.1744.

LEOPOLDINO, K. D. M. et al. Creativity techniques: a systematic literature review. Product: Management \& Development, v. 14, n. 2, p. 95-100, 2016. http://dx.doi. org/10.4322/pmd.2016.015.

LU, Y. Industry 4.0: A survey on technologies, applications and open research issues. Journal of Industrial Information Integration, p. 1-10, 2017. http://dx.doi.org/10.1016/j. jii.2017.04.005.

NOY, N. F.; MACGUINNESS, D. L. Ontology development 101: a guide to creating your first ontology. Stanford: Stanford University, 2001.

NUÑEZ, D. L.; BORSATO, M. An ontology-based model for prognostics and health management of machines. Journal of Industrial Information Integration, v. 6, p. 33-46, 2017. http://dx.doi.org/10.1016/j.jii.2017.02.006.

PAHL, G.; BEITZ, W. Engineering design: a systematic approach. Edited by WALLACE, K. Título. 2nd ed. Darmstadt, Germany: USA: Springer Science \& Business Media, 2013.

PEFFERS, K. et al. A design science research methodology for information systems research. Journal of Management Information Systems, v. 24, n. 3, p. 45-77, 2007. http:// dx.doi.org/10.2753/MIS0742-1222240302.
SAUNDERS, M. et al. Research methods for business students. London: Pearson Education Limited, 2012.

SERRA, I.; GIRARDI, R.; NOVAIS, P. Evaluating techniques for learning non-taxonomic relationships of ontologies from text. Expert Systems with Applications, v. 41, n. 11, p. 5201-5211, 2014. http://dx.doi.org/10.1016/j. eswa.2014.02.042.

SHROUF, F.; ORDIERES, J.; MIRAGLIOTTA, G. Smart factories in Industry 4.0: a review of the concept and of energy management approached in production based on the Internet of Things paradigm. In: INTERNATIONAL CONFERENCE ON INDUSTRIAL ENGINEERING AND ENGINEERING MANAGEMENT, 2014, USA. Proceedings... USA: IEEE, 2014. p. 697-701. http://dx.doi. org/10.1109/IEEM.2014.7058728.

SIRIN, E. et al. Pellet: a practical OWL-DL reasoner. Journal of Web Semantics, v. 5, n. 2, p. 51-53, 2007. http://dx.doi. org/10.1016/j.websem.2007.03.004.

SIRIN, E.; PARSIA, B. SPARQL-DL: SPARQL query for OWL-DL. In: CEUR WORKSHOP, 2007, Cidade. Proceedings... Berlin: Springer, 2007.

STAAB, S.; STUDER, R. Handbook on ontologies. In: STAAB, S.; STUDER, R. (Ed.). Handbook on ontologies. 2nd ed. Berlin: Springer Berlin Heidelberg, 2009. http:// dx.doi.org/10.1007/978-3-540-92673-3.

STANFORD CENTER FOR BIOMEDICAL INFORMATICS RESEARCH. PROTÉGÉ: versão 5.2.0. Stanford: Stanford University, 2017. Available from: <https://protege.stanford. edu/>. Access in: 13 Dec. 2019.

TANASIJEVIC, M. et al. A fuzzy-based decision support model for effectiveness evaluation - a case study of examination of bulldozers. International Journal of Industrial Engineering: Theory, Applications and Practice, v. 26, n. 6, 2019.

TRAPPEY, A. J. C. et al. A review of essential standards and patent landscapes for the Internet of things: a key enabler for industry 4.0. Advanced Engineering Informatics, v. 33, p. 208-229, 2017. http://dx.doi.org/10.1016/j.aei.2016.11.007.

WAGHMODE, L. Y.; PATIL, R. B. Reliability analysis and life cycle cost optimization: a case study from Indian industry. International Journal of Quality \& Reliability Management, v. 33, n. 3, p. 414-429, 2016. http://dx.doi. org/10.1108/IJQRM-11-2014-0184.

YANG, L. et al. Design-for-reliability implementation in microelectronics packaging development. Microelectronics International, v. 28, n. 1, p. 29-40, 2011. http://dx.doi. org/10.1108/13565361111097092.

ZHONG, R. Y. et al. Intelligent manufacturing in the context of industry 4.0: a review. Engineering. Elsevier Ltd, v. 3, n. 5, p. 616-630, 2017. http://dx.doi.org/10.1016/J. ENG.2017.05.015. 